\title{
International standard reagents for HPV detection $^{1}$
}

\author{
Sonia R. Pagliusi ${ }^{\mathrm{a}, *}$ and Suzanne M. Garland ${ }^{\mathrm{b}, \mathrm{c}}$ \\ ${ }^{a}$ Department of Immunizations, Vaccines and Biologicals, World Health Organization, Av Appia 20, 1211 Geneva \\ 27, Switzerland \\ ${ }^{\mathrm{b}}$ Department of Microbiology and Infectious Diseases, The Royal Women's Hospital, 132 Grattan Street, \\ Melbourne, Vic., Australia \\ ${ }^{\mathrm{c}}$ Department of Obstetrics and Gynaecology, University of Melbourne, Vic., Australia
}

\begin{abstract}
Humam papillomavirus is the commonest genital viral infection in healthy sexually active subjects, and the presence of chronic or persistent HPV types in genital cells may constitute a prognostic marker of underlying, or predict future HPVassociated diseases. A variety of novel tests for detecting the presence of oncogenic HPV types in biological specimens have been reported. These are based on the various stages of infection and viral life cycle. HPV infects squamous epithelium with expression of various gene products intimately linked to epithelial cell differentiation. Hence, there are basically three classes of detectable markers directly derived from HPVs: molecular markers based on detection of nucleic acid sequences, serological markers based on detection of antibodies against viral proteins, and cellular markers based on detection of proteins expressed intracellularly, upon either infection or carcinogenesis. The nature of various assays and the development of international standard reagents for qualitative and quantitative assessment of assay performance are outlined. There is an increasing demand to develop standard tools to assess the quality of HPV detection systems, for regulatory and clinical management purposes. International standard reagents for HPV will help defining the analytical sensitivity and specificity of various detection methods, and will allow assuring that laboratory services used to evaluate disease burden, HPV vaccines, and cancer prevention strategies are accurate and comparable worldwide. The advancement of prophylactic vaccine candidates against HPV infections and related diseases stresses the increasing importance of HPV assays in monitoring the impact of HPV vaccination on disease burden.
\end{abstract}

Keywords: Papillomavirus, cervical cancer, diagnostic, genital infection, hybridization, PCR, serology, vaccine

\section{Introduction}

Papillomaviruses are a large family of non-enveloped DNA viruses that within the animal kingdom cause a variety of epithelial lesions, ranging from benign warts to invasive cancers. Of the known human papilomaviruses (HPV) associated cancers [19], cancer of the uterine cervix accounts for $90 \%$, whilst other anothenital cancers (vulvae, vagina, penis, anus) for $5 \%$,

\footnotetext{
${ }^{1}$ The authors only are responsible for the contents of the publication.

*Corresponding author: Dr Sonia R. Pagliusi, Immunization, Vaccines and Biologicals, Family and Community Health, World Health Organization, Avenue Appia 20, 1211 Geneva 27, Switzerland. Tel. +41 22791 4265; Fax: +41 22791 4860; E-mail: pagliusi@who.int.
}

with cancers of head and neck corresponding to about $1 \%$ [44].

Over the past decades, prevention programmes for cancer of the cervix have been essentially based on the detection and treatment of precursor lesions (highgrade cervical dysplasia) or early cancer by screening women at risk using the cytological method described by Papanicolau, otherwise known as the "Pap smear" [43]. Despite great progress in cancer control through such cervical cytology screening programmes, high coverage and quality control and assurance are crucial in achieving significant reduction in disease burden [13]. However in low resource countries appropriate resources and continuous quality assurance programmes are not always available. In addition, it is recognized that the traditional Pap smear tests can give 
both false positive as well as false negative results in the screening for precursor lesions and/or cancer. Hence, the scientific community has been actively in search of effective technologies to complement and improve cytology based cervical screening programs.

The causal relationship between HPV infections and cancer of the uterine cervix was first suggested in the 1970's [16], and comprehensively confirmed later, in finding that virtually all cases studied worldwide have at least one of 15 high risk oncogenic types of HPV [63]. Hence, the presence of oncogenic HPV types in cervical epithelium, is recognized as a necessary cause for triggering cancer of the cervix in women. That is, women will not develop cancer of the cervix without a pre-existing infection with the oncogenic HPVs. The corollary however it is not true, as cancer is a rare outcome of this very common viral infection. Indeed the majority of HPV infections are transient, being cleared by immune surveillance usually within one or two years [18]. HPV was also being identified as the aetiological agent of other cancers, namely $70 \%$ of anal cancers, $50 \%$ of vaginal cancers, and about $25 \%$ of head and neck squamous cell carcinomas (HNSCC) contain HPV DNA, mostly type 16 [54].

The natural history of HPV infection is such that the lifetime risk for development of cervical cancer without involvement of any screening programme is in the order of $3 \%$ and with annual Pap smears, less than $1 \%$. The success of screening programmes relates in part from the fact that cervical cancer takes several decades to develop from chronic oncogenic HPV infection [1]. Although HPV is the commonest genital viral infection in healthy sexually active subjects, the presence of persistent oncogenic HPV types in tissue samples may constitute a prognostic marker of underlying or predict disease in the future. The corollary however is not true; the presence of HPV DNA alone does not necessarily imply underlying disease.

\subsection{Background on the cell biology of HPV}

HPV infects squamous epithelium with expression of various gene products intimately linked to epithelial cell differentiation. HPV infection does not cause a systemic infection; it does not kill the keratinocyte and it induces no or a poor, slow, local inflammatory response. Humoral immunity does not appear to be as important as cell-mediated immunity in clearing HPV infection, but rather is important in protecting an individual from becoming infected. Of note, in natural infection, only around $50 \%$ of those becoming HPV DNA positive show a systemic neutralizing antibody response. The remainder of individuals clear infection without developing a measurable antibody response [2].

Following infection of basal epithelial cells, the viral genome is maintained as a low-copy number episome in cells of the basal and parabasal layers and is amplified as the infected cell differentiates and migrates towards the epithelial surface. It is only in the most superficial layers of the epithelium, that late genes coding for viral capsid proteins (L1 and L2) are expressed and the amplified genomes then packaged into infectious virions, with release at the epithelial surface during cellular desquamation [32]. It is because of this complex interaction, that HPV cannot be propagated in vitro in cell lines, as many other viruses that are cultured for diagnostic purposes. However viral nucleic acid can be detected as episomal genomes by various molecular hybridization assays. It appears from some studies that a productively infected cell expresses early viral proteins E4, E6, E7 during its migration from the basal layer to the epithelial surface [35]. Expression of the major capsid protein L1 appears to follow expression of E4, and is detectable in cells approaching the epithelial surface. During the progression from productive infection to cellular transformation leading to cervical cancer, the viral DNA becomes integrated into the host cell genome (often with large deletions in the L1gene), and loses the ability to produce infectious virions. Productive HPV infection is manifest as changes cytologically /histologically identified as epithelial lesions. If cell mediated immunity fails to induce lesion regression and viral clearance, then persistent infection may result. In low-grade cervical intraepithelial neoplastic (CIN1) lesions all early proteins are expressed, the lesions are genetically stable and regress in around $60 \%$ of cases within time, with an appropriate host immune response (median duration of CIN1/LSIL is 6 months), or do remain unchanged for years [42]. In many highgrade cervical intraepithelial neoplastic lesions (CIN 2 and 3) and in cancers however, viral gene expression is dysregulated, with constitutive expression of the early oncogenes E6 and E7 essential for progression to, and maintenance of malignancy. The histological changes from normal epithelium to cervical intraepithelial neoplasias [CIN] grade 1 to 3, following HPV infection, is schematically shown in the cartoon (Fig. 1).

With this knowledge, novel diagnostic tools for detecting the presence of oncogenic HPV DNA or the effects in cervical samples, have been intensively studied. There are basically three classes of detectable markers directly deriving from HPV viruses: Molecular mark- 


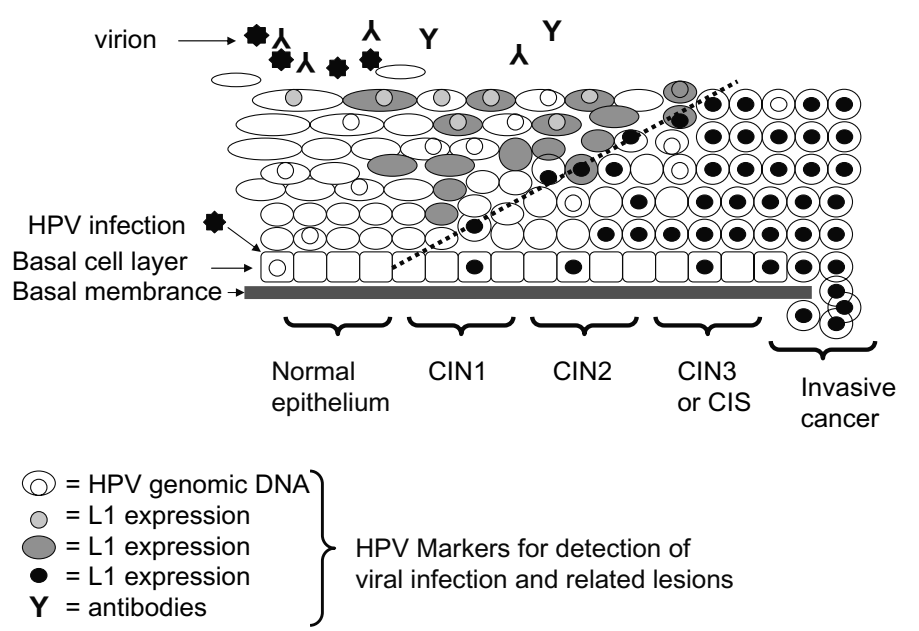

Fig. 1. Schematic representation ofhistological changes followingHPV infection and carcinogenesis. Oncogenic HPV types initiate a productive infection in the basal cells, with detectable expression ofviral DNA, E4,E7, proteins, and L1 expression and virion production and release in the superficial cell layers. E4/L1 expressingcells form low grade lesions. Over time, integrated viral genes express oncoproteins (E6/7) leading to uncontrolled cell growth, to form high grade lesions and invasion.

ers based on detection of viral nucleic acid sequences, serological markers based on immunological responses such as antibodies, and host cellular markers based on proteins expressed upon infection and during oncogenesis. The nature of various assays to detect these three distinct markers of HPV infections and related diseases, their standardization, and potential applications are outlined below.

Because carcinogenesis is triggered by this infectious agent, it has been possible to develop prophylactic vaccine candidates to prevent infection, its related diseases, especially life-threatening cancers [12]. The prophylactic HPV vaccines may have implications not only in the prevention of cancer of the cervix, but also of a substantial fraction of other ano-genital cancers, and potentially head and neck squamous cell carcinomas [45]. The applications of HPV detection methods with respect to vaccines evaluation is discussed below.

Regression of lesions is accompanied by a CD4 T cell dependent delayed type hypersensitivity response. Cell mediated assays are currently used for assessment of HPV induced immune responses upon infections, disease, and vaccination. The methods include mostly proliferation assays, cytokine release assays (ELISPOT or cell culture supernatant), and in vivo delayed type hypersensitivity (DTH) assays. Given the many clinical trials assessing therapeutic effects of vaccination with HPV antigens, it would be desirable to have standard reagents for use in assays determining cell-mediated responses. It was suggested to develop and evaluate standard positive control reagents (substrates) for cell- mediated immunity assays [70]. However, we will focus here only on markers specific and directly derived from HPV-expressed molecules. T-cell based assays may not be appropriate for characterization of prophylactic vaccine responses.

\subsection{HPV detection methods and standard reagents}

Clinical diagnostic is characterized by the application of methods to a clinical activity, allowing clinicians to extract valuable information from biological samples submitted for various assays and to make a definitive diagnosis. For this purpose available tests need to be validated, i.e. determine their capacity to correctly classify an individual within his/her health/disease status.

International standard reagents may assist in the qualitative and quantitative assessment of diagnostic tests, to evaluate the performance and the clinical benefit of various detection methods. Since 1950 the World Health Organization (WHO), as part of its constitutional mandate, develops international standards for a wide range of biological substances used for medical purposes. Over the past years, WHO has worked with the scientific community, national regulatory authorities, other standards-setting bodies, and users, through a series of consultations to review the scientific basis of biological products, and develop reference materials or reagents. The published catalogue of WHO biological reference materials includes todate over 300 materials (a list can be found at www.who.int/biologicals). Re- 
cently, efforts were directed towards developing specific standard reagents to detect HPV viral genes, their expressed proteins, or their elicited antibody responses [60].

An international standard reagent is a preparation to which an international unit of activity has been assigned, that is traceable to a defined part of the content of an ampoule. International standards must fulfill several criteria, including demonstrating consistent performance, demonstrating long-term stability under selected storage conditions, and being able to perform in a variety of detection assays. Such standards are intended to make estimations of concentration of molecules or potency, of an appropriate test sample, by direct comparison in suitable biological test systems.

\section{HPV DNA detection methods, standards and applications}

HPV DNA can be detected in biological material containing infected epithelial cells, mostly derived from scrapes from the ectocervix or other genital tract epithelial sites. In laboratory based measurements of HPV DNA we need to distinguish analytical sensitivity and specificity from clinical sensitivity and specificity. The term analytical sensitivity refers to the number of HPV-viral genomes that are identified in a sample by a given test or assay, expressed as a given virus amount of analyte or molecules, whereas analytical specificity refers to the genotypes of HPV that are correctly identified by a test result. In contrast, the clinical sensitivity refers to the proportion of women with disease, identified by a positive test result, whereas clinical specificity indicates the proportion of positive results that correlate to underlying disease [59]. Here we will discuss mostly the analytical sensitivity and specificity of methods, rather than their clinical aspects.

\subsection{Methods of HPV DNA detection: Hybrid capture and amplification}

Currently two types of amplification assays are used in determining HPV DNA. One is a target amplification , polymerase chain reaction (PCR) and the other signal amplification following DNA and RNA hybridization and detection by Hybrid Capture 2 (HC2), (Digene Corporation, Gaithersburg, Maryland, USA) [31].

The HC 2 test is the only FDA approved test so far and is used to show the presence or absence of HPV DNA in exfoliated cells from a woman's cervix, usually obtained by gently scraping the surface of the cervix. This assay is based on the principle that HPV DNA isolated from biological specimens, hybridizes in solution with long synthetic RNA probes complementary to the genomic sequences of specific HPV types. The test is currently provided as two available RNA-probe mixtures containing either five low risk (LR) types $(6$, $11,42,43,44)$ or 13 high risk (HR) types $(16,18,31$, $33,35,39,45,51,52,56,58,59$ and 68). To reduce time and cost, most laboratories only use the HR probe mixture, being the relevant probe mix for clinical management. The HC2 provides a semi-quantitative measure of viral load, as measured by the intensity of emitted light, which is proportional to the amount of target DNA present in the specimen. The relative analytical sensitivity of the assay is equivalent to $1 \mathrm{pg}$ of HPV DNA (5000 copies of HPV genome), although the HR probe cocktail may cross-react with HPVs not in the probe mixture (i.e. types 53, 66, 67, 73). Anything below that level indicates either the absence of HPV or HPV-amounts are below the detection level. A new rapid test under development by Digene detects HPV DNA, and processes 10-20 samples in one batch within two hours. The sensitivity of this test is comparable to the currently available HC 2. A chemiluminescent substrate is used and signal output can be read by a Luminometer readout at a central laboratory, and may be relevant for use in field conditions, health enters or clinical practice.

PCR is a selective amplification assay, increasing exponentially and reproducibly the target HPV sequences present in biological specimens, and theoretically capable of producing one billion copies from a single double stranded DNA molecule, following 30 cycles of amplification. The sensitivity and specificity of PCR-based methods can vary, depending on the primer sets chosen, the size of the PCR product, reaction conditions and performance of the polymerase enzymes used in the reaction, the spectrum of HPV DNA amplified and ability to detect multiple types [20]. Most primer sets used for HPV-PCR are designed to target the L1 gene, but primers targeting the oncogenes E6 and E7 have also been used in clinical studies [28]. The choice of the viral genomic sequences used for PCR has implications for analytical sensitivity, and hence HPV screening strategies in the clinical diagnosis and management of cervical cancer. Generally, the analytical sensitivity of PCR based methods is higher than hybridization assays only. Most accurate PCR methods can detect the equivalent of 1-10 copies of viral genome per reaction, although the results achieved using the same methods 
can vary depending on factors intrinsic to laboratory individual performance, which may be linked to handling of samples and reagents [47]. Following PCR, distinction of HPV types can be achieved by sequencing or hybridization with type-specific oligonucleotide probes using various detection methods, including dot blot, microtitre plate, reverse line blot strip and microchip formats.

Recently, Roche Diagnostics (Indianapolis, IN, USA) have developed an HPV ELISA based AMPLICOR HPV kit with CE marking ("Conformité Européene") for in-vitro diagnostics and is the first commercially available PCR-based test for the detection of HPV. This new test, identifies the same13 high-risk genotypes of HPV as the HC2 test, and will allow diagnostic laboratories to detect HPV DNA by PCR, although not to discriminate genotypes [36]. The "Linear array ${ }^{\circledR}$ " assay, also developed by Roche, allows detection of 37 different HPV genotypes. It detects amplified type specific HPV DNA with oligonucleotides immobilized onto a nylon membrane, an easy to perform detection method. The advantages of this method are speed, with a turn around time of 1 day, plus ease in interpreting individual and/or multiple genotypes, yet without requiring expensive, specialized instrumentation [27].

The development of a quantitative and sensitive hightroughput procedure for the identification of multiple high and low-risk genital HPV genotypes in a single reaction has been recently reported as multiplex papillomavirus genotyping (MPG) [53]. MPG is based on the amplification of HVP DNA by a general primer PCR $(\mathrm{GP} 5+/ 6+)$ and the subsequent detection of the products with type-specific oligoucleotide probes coupled to fluorescence-labeled polystyrene beads (luminex suspension array technology). Theoretically, MPG can detect up to 100 different HPV types simultaneously, and appears to be suitable for large scale epidemiological studies and vaccination trials, as well as for routine diagnostic purposes, though it remains to be validated.

A novel HPV oligonucleotide microarray has been developed (Biomedlab, Seoul, Korea), which allows for detection of $22 \mathrm{HPV}$ types using an aldehydederivatized slide glass. PCR products are generated in the presence of fluorescein-tagged nucleotides and hybridized onto the chip and scanned by laser fluorescence, thus able to detect multiple infections with one hybridization step [55]. Ideally, a larger number of HPV type-specific oligonucleotides could be spotted on the Chip, although this method requires the presence of expensive equipment and may not be suitable for many laboratories. Utility of real-time and quantitative PCR in detection of HPV has also been investigated in a number of studies and can provide tools for ready quantitation of various HPV genotypes in specimens [22].

A test for identification of mRNA encoding HPV oncogenes (PreTect HPV-Proofer, Norchip, Norway) intended for diagnostic purposes has been described [30]. E6/E7 mRNA will be present when the virus is actively transcribing and producing oncogene proteins, thus the test is designed to detect RNAs from the E6 and E7 region of the viral genome. Larger scale, population based studies are necessary to evaluate the predictive values of this HPV test.

Finally, a protocol for the amplification of papillomavirus oncogene transcripts (APOT) from cervical specimens has been proposed that allows the distinction of episomal from integrated HPV mRNAs [26]. In most cervical carcinomas, human papillomavirus (HPV) genomes are integrated into host cell chromosomes, such that transcribed mRNAs encompass viral and cellular sequences. In contrast, in early preneoplastic lesions, HPV genomes persist as episomes, and derived transcripts contain exclusively viral sequences. Thus, detection of integrated-derived HPV transcripts in cervical swabs or biopsy specimens by the APOT assay points to advanced dysplasia or invasive cervical cancer. However, because the assay is based on RTPCR protocols, sequencing steps, and is type specific it has not been adapted readily to use in the routine diagnostic testing.

With a variety of assays for HPV DNA detection, and new and improved methodologies under way, there is increasing demand to standardize the measurement tools to assess the performance of molecular tests and methodologies, for both regulatory and clinical management purposes.

\subsection{Standards for HPV DNA reagents}

The goal of accurate detection of infectious agents is to provide consistent and meaningful results in the research and clinical setting to help target and focus resources in disease prevention and control. An intrinsic part of using such technology should be the use of well-characterized standards. To assess the quality of molecular detection methods, international standard reagents are already widely used for several other viruses including hepatitis $\mathrm{A}, \mathrm{B}$, and $\mathrm{C}$ and human immunodeficiency virus (HIV) [21,49,51]. Ideally standard reagents should mimic properties of biological samples under measurement and, allow evaluation of the full 
laboratory sample processing procedures. It would be desirable that HPV DNA standards should contain full HPV genomes to allow identification of any genomic region to be targeted in detection assays, and should be presented in a human genomic background.

Historically, international standards in the form of nucleic acids have been isolated from pools of biological material infected with the virus of interest [71]. As HPV is not a plasma derived virus, there is no such abundant source of biological material to generate HPV DNA standard reagents. Epithelial cells derived from cervical scrapes or small genital biopsy specimens, obtained for diagnosis, provide only low numbers of HPV genomes and often harbor multiple HPV types.

Therefore recombinant plasmids representing double-stranded full genomic, cloned DNA sequences of HPV types were proposed as standards for HPV detection [69]. Such material is renewable and hence readily available. An international collaborative study demonstrated the feasibility of this approach using a variety of HPV DNA detection assays, and the results of this study support the concept that recombinant HPV DNA constructs can be used to develop international standard reagents [47]. The international collaborative study group recommended that the priority for international standard reagents be for HR types HPV 16 and 18 as related to around $70 \%$ cancer cases, and then expand to the next most prevalent HR HPV types: HPV types $31,33,35,45,52$, and 58 [6]. For HPV DNA, it is desirable to develop monovalent or individual HPV type standards, allowing unequivocal calibration of individual HPV DNA material and assessment of potential interference when multiple HPV types are present [60].

PCR primers directed at the E6 or E7 regions, encoding oncogenic proteins, have been described as preferable, whereas those targeting the L1 or E1 region may miss advanced disease, because the L1/E1 regions are often lost during integration of viral DNA into host genomic DNA [28]. The plasmid materials proposed by WHO to be developed as international standard reagents contain the full genome sequence of HPV virus types and could be useful in tests targeting both late and early viral genes.

Although such standards are not widely available yet, preliminary studies are being performed to verify material stability and reliability, including titration of the plasmid, as well as stability of the plasmid during a freeze-dry process. In addition, it may be best to designate international standard reagents using arbitrary international units, and the HPV DNA international standard unit remains to be established. Future studies need to determine if HPV DNA candidate standards can also assess the full spectrum of sample processing schemes that would mimic diagnostic sample preparation including DNA extraction, precipitation, or centrifugation procedures.

\subsection{Applications of HPV DNA detection assays}

The applications of HPV DNA standard reagents will vary depending on the setting in which they are used. Detection assays for HPVDNA with high analytical sensitivity have been crucial in demonstrating the aetiological role of oncogenic HPV presence in cervical cancer [37]. Similary, in prevalence surveys for HPV related cancer cases, as well as among general population, the analytical sensitivity of genotyping assays must be set for accurate discrimination of differences in the distribution of viral types in various geographical areas [5]. Methods with high analytical sensitivity are crucial in order to accurately evaluate that vaccine candidates are preventing the viral infections, and related diseases. In clinical trials of prophylactic or therapeutic vaccines for example, molecular detection methods based on PCR are proven useful in monitoring the efficacy of prophylactic vaccination [14,33,61]. Moreover, it is expected that upon vaccination, a reduction in the prevalence of the types included in vaccine preparations should be measurable in vaccinated cohorts. Sensitive type specific assays are therefore key in the evaluation of vaccination impact on prevalence of vaccine related genotypes, or potential genoptype replacement in post-vaccination periods.

In contrast, the use of HPV testing as a tool for cervical cancer screening programmes, needs to be thoroughly considered in a context of diagnostic algorithms, and may have to take into account the costeffectiveness and the long-term benefits of such programmes. Detection of oncogenic HPV DNA is considered a predictor of underlying high grade dysplasia of the uterine cervix, at risk for potential invasive disease, but may depend on other factors such as age, viral load [74], other concomitant genital infections [58], high parity and environmental factors [3,7]. Therefore, algorithms of care including HPV testing are being developed and have been endorsed by various expert groups (IARC, ACOG, NHMRC) for use in different combinations and situations adapted locally. These applications include the option of HPV DNA testing with or without Pap, triage of ASCUS/border line lesions, and/or of treatment for cervical dysplasia as reliable predictor for residual disease $[8,72]$. With the appreci- 
ation of the role of HPVs in cervical cancer, the American College of Obstetricians and Gynecologists recently published a new guideline for cytology screening of cervical samples that incorporates the use of HPV detection in women $\geqslant 30$ years of age as an important adjunct [73].

Most of the published studies to date have been conducted utilizing HC2, with studies based on PCR detection of HPV DNA only just coming to light. In the recently published ALTS study when HPV genotyping was evaluated, authors concluded that ASCUS and LSIL patients who have a positive HPV16 result by genotyping are at significantly greater risk for detection of high-grade abnormalities during a 2-year followup period when compared to those who test positive for another oncogenic type of HPV or who are HPVnegative [4]. Furthermore, HPV DNA testing, in particular PCR, can be performed on self-collected samples; these have similar sensitivity to clinician-collected samples [72]. Finally, application of HPV DNA testing may be useful as a rapid intermediate end point for monitoring the efficacy of surgical treatment of CIN usually results in clearance of HPV infection [10].

Despite their high clinical sensitivity for high grade cervical intraepithelial neoplasias, the clinical specificity of HPV DNA tests that have been applied in longitudinal studies is not ideal: It has been hypothesized that some HPV DNA detection methods, with very high analytical sensitivity, will show a higher positivity rate in women that will not be at risk for developing disease and may increase the number of referrals. Hence the clinical management of genital HPV related diseases has suggested that less sensitive HPV assays may be appropriate [59]. Conversely, an HPV test with too low analytical sensitivity will miss individuals positive for the viral DNA, thus at risk. A balance must be found, and viral load threasholds must be defined for HPV tests to be used in screening programs, triage of abnormal cytology, or for post-treatment monitoring. In practical terms, a definitive diagnosis should rely on the combined results of several tests, including histopathology if possible.

\section{HPV antibodies detection methods, standards and applications}

Multiple serological assays for HPV antibodies have been developed such as enzyme-linked immunoabsorbent assay [ELISA], including sandwich ELISA and radio immuno assays (RIA), competitive ELISA, neu- tralization and pseudovirion based in vitro neutralization methods.

Practically, HPV infections will result in detectable antibodies in only $50-60 \%$ of sera from individuals with HPV DNA infection [2]. Up to half of women with cervical cancer have at diagnosis sera antibodies to the HPV oncoproteins E6 and E7, which are rarely found among healthy women; however these are not validated markers of occult cervical cancer [29]. AntiHPV antibodies are not only detected in serum but also in other body fluids, such as oral and vaginal lavage [38, 39]. They may be considered in some circumstances markers of disease, as for instance $\operatorname{IgA}$ antibodies to HPV in oral fluid were also reported to correlate with cervical disease [34]. However the diagnostic value of detection of HPV specific antibodies in body fluids other than serum, to determine HPV immune responses, remains to be established.

\subsection{Methods of HPV antibody detection: HPV ELISA assays and neutralization assays}

Antibody detection methods are mostly directed to antibodies produced against the major capsid protein $\mathrm{L} 1$, often released upon productive viral infections. The antibody based methodology has so far shown insufficient sensitivity, complicated by determination of an appropriate cut-off, resulting in low specificity. HPV VLP-based ELISA assays to L1 are type-restricted measures of present or past genital HPV infections. The biological relevance of immune responses should correlate to neutralizing antibodies. These can be assessed in sera of subjects either by some type specific ELISA or biological assay systems able to recognize neutralizing epitopes of HPV.

HPV capsids are composed of two proteins, the major capsid protein L1 and the minor capsid protein L2 [50]. L1 protein when expressed in eurakyotic cells, has the capacity to spontaneously form the three dimensional structure necessary to aggregate as pentameric structures, also called capsomers [40], and finally to self assemble into VLPs that are similar to authentic virions, and can induce high titers of antibodies that neutralize papillomavirus infection in vitro [24]. Both capsomers and capsids have been used as antigenic components for vaccination, as well as substrates for immunoassays (Fig. 2).

Most assays depend on the conformation of the antigen used as substrate for antibody detection. Some assays rely on VLPs, and these prove to be necessarily of high purity and quality, and are consequently expensive 


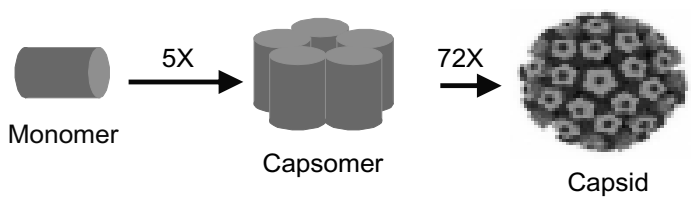

Fig. 2. Schematic representation of monomeric recombinant L1 proteins that self-assembly in pentameric structures as capsomers, and subsequently aggregate to form virus like particles (VLPs), similar to empty viral capsids (courtesy of Dr John Nieland).

to produce. The use of capsomers, which give conformational antigens in the form of pentamers of the viral capsid proteins, is being studied as an alternative. Additionally, assays detecting antibodies to the early proteins E6 and E7 have been also described [29].

Approaches to improve the assay have been the inclusion of Heparin to the ELISA plates, to capture properly folded L1 [64], or the use of an alternative antigen, such as the Glutathione S-transferase (GST)-L1 fusion proteins [54]. These antigens are used in a multiplex bead assay and allow for the detection of antibodies against multiple types [65], though non-specific background remains an issue [66]. Characterization of the assay has been limited and $20 \%$ differences have been reported between the VLP and GST assays.

A human papillomavirus (HPV) multiplexed competitive Luminex immunoassay first described by Opalka and colleagues [41], was optimized to increase both the analytical sensitivity and the clinical specificity of the assay [9], to more effectively discriminate the lowtiter antibody response of HPV-infected persons from non-infected individuals. The new version is based on conjugation of VLPs to microspheres and the use of specific monoclonal antibody to neutralizing epitopes of viral particles. The assay was automated by use of a TECAN Genesis Workstation, thus improving assay throughput, reproducibility, and operator safety. The assay was validated then using several distinct serum panels from individuals known to be at low and high risk for HPV infection. This high-throughput assay has proven useful for performing epidemiology studies and evaluating the efficacy of prophylactic HPV vaccines.

Previously available HPV neutralization assays were insensitive, cumbersome and/or time-consuming [48]. A surrogate assay relies on hemaglutination inhibition, based on the observation that VLPs crosslink mouse red blood cells that could be reduced or abolished in the presence of an inhibiting L1-specific antibody. The disadvantage of the hemaglutination inhibition assay is that only a subset of neutralizing antibodies prevents agglutination. A similar limitation applies in the case of competitive radioimmunoassay and the multiplex assay, as only a subset of antibodies is able to compete in the assay.

Recently, a simple and high throughput pseudovirusbased in-vitro neutralization assay was developed [46]. Neutralization is monitored by measuring the activity of secreted alkaline phosphatase (SEAP), which is expressed from a SEAP gene that is included in the pseudovirus as a reporter gene. The approach is easy to scale up, as pseudovirus stocks are not a limiting step. The SEAP neutralization and VLP-ELISA assays have comparable sensitivity for detecting capsid antibodies generated after natural HPV infections and chemiluminescent detection improves the dynamic range of the assay sensitivity. The SEAP assay appears more specific in detecting type-restricted antibody responses to VLP vaccination, and available data suggest good interlaboratory reproducibility. The limitations of SEAP neutralization assay are that there may be non-specific inhibition at low sera-dilutions, it requires cell culture, and chemiluminescent reagents are expensive.

\subsection{Standards for HPV antibody detection}

Following natural infections with HPV, antibodies responses are usually of relatively low titers. In contrast, following vaccination with prophylactic viral like particles (VLPs), circulating neutralizing antibodies appear to be the basis for protection against vaccine related HPV infectious and disease. Hence, type-specific antibody detection will play a key role in determining efficacy, quality, potency and duration of immune responses to HPV vaccination and eventually correlates of protection.

Standard reagents based on antibodies are intended for use in the estimation of potency of an appropriate test sample, by direct comparison in suitable biological test systems, and are usually established in the context of international collaborative studies. In order to develop standards for HPV antibody detection, the World Health Organization has coordinated an international collaborative study among laboratories implicated either in clinical studies or vaccine development [11]. Briefly, the study was aimed to assess the specificity and sensitivity of assays currently in use for measuring antibodies to the major viral capsid protein L1 to HPV, and to determine whether the use a standard reagent and the expression of titers in identical arbitrary units would improve reproducibility of results in various laboratories . Further, we assessed whether the expression of the titers in identical arbitrary units would improve 
comparability of results between laboratories. Samples studied included sera from individuals naturally infected with HPV 6/11, 16 and 18, a negative serum from a virgin woman, and sera from recipients of HPV vaccine candidates under clinical development, in a total of 12 samples.

The study showed that the range of titers for HPV 16 antibodies in assays expressed by the participating laboratories varied up to 25 fold for one sample. However, when the expression of HPV 16 antibody concentration in each sample was calculated relative to a single arbitrary serum sample assigned the unitage of " 1 " (one), the comparability of measurements was improved between laboratories, reducing the variability range to 2 to 3 fold. Therefore, the results of the collaborative study suggest that establishment of International Standard reagents for antibodies to HPV would facilitate the comparison of HPV antibody measurements between assays and laboratories worldwide.

Up to half of women with cervical cancer have been reported to show at diagnosis, sera antibodies to the HPV oncoproteins E6 and E7, which are rarely found among healthy women [57]. However, standard reagents for measuring antibody responses to other HPV proteins such as L2, E6 or E7, have not been assessed by an international collaborative study so far. In addition, operating procedures and quality control of the assays have not been fully resolved and standardized [11].

\subsection{Applications of HPV antibody standard reagents}

HPV standard reagents for serology will be crucial for improving the sensitivity of antibody assays, the consistency of results worldwide, as well as improving inter-laboratory and inter-assays variability, as suggested in comparative studies [11] and thus have a number of applications, as their use would improve assays performance, allow valid comparison of results, among laboratories, and facilitate monitoring of vaccine effectiveness.

There is currently no single "gold standard" for measuring the HPV serological responses, and different assays may be preferable depending on the questions being asked. The presence of anti-HPV antibodies in sera of individuals is mostly a marker for previous infections, not necessarily correlating to present infection or disease, as serological evidence for the association between HPV 16 infection and carcinomas is weak. In addition, HPV seropositivity may result from previous viral infections that have been efficiently cleared or may be present as asymptomatic infections that deserve no clinical management. Consequently, serological assays have not proven their clinical diagnostic value thus far.

Nevertheless, HPV serology has proven valuable in epidemiological studies based on seropositivity for HPV, in understanding the natural history of infections and cancer. Importantly, antibody based immune responses to HPV using various methods has contributed notably to understanding the protection conferred by neutralizing antibodies and allowed important advancements in the area of prophylactic vaccine development, as well as their evaluation by ELISA [15].

In the context of vaccination, serological assays are used to screen volunteers for vaccine trials as to previous exposure to HPV, and evaluating seroconversion in vaccinees. Moreover, the use of international standard reagents in the quality assurance of future recombinant VLP-based HPV vaccines, may be valuable in potency assays used for lot release, if immunogenicity assays are used for this purpose.In post vaccination studies monitoring vaccination performance (immunogenicity) and vaccination coverage, and surveillance of correlates of protection in vaccinated cohorts, are potential areas for using antibody based measurements in the near future.

In conclusion, for assessment of cumulative exposure to genital HPV infection prior to vaccination, the preferred assay to use may be the VLP ELISA, since it is the best validated assay, easier and less expensive to conduct than the pseudovirus assay. However, the availability of high quality VLPs may be a limiting factor. The SEAP assay measures all classes of antibodies relevant to protection, is type-restricted in measuring VLP-induced antibodies, is relatively simple and high throughput, and therefore could be used to monitor vaccine performance in central laboratories or locally after shipment/storage of sera. It might be particularly useful in the attempt to study immune correlates of protection.

\section{Detection of HPV derived proteins}

The viral proteins E6 and E7, which are selectively expressed in HPV-associated cancers, display potent transforming and tumourigenic activities in tissue culture and transgenic mouse models, respectively. Of particular importance is their ability to promote genetic instability through induction of cellular proliferation, disruption of cell-cycle checkpoints, inhibition of 
apoptosis and induction of telomerase. For oncogenesis to occur, host immune surveillance, intracellular specific cell signalling cascade regulation, and paracrine regulation of viral gene activity must be breached [17]. Based on this concept, additional biomarkers have been proposed that may be useful in identifying precancerous lesions at risk for malignant progression, instead of regression. Such markers identified recently are, for example, the human cyclin-dependent kinase inhibitor, p16INKa4, the retinoblastoma protein $(\mathrm{Rb})$, the p53 protein, the KI-67 [23], the minichromosome maintainance protein (MCM), and cyclins, which are not usually expressed above the basal layers in normal epithelium [35]. Potential proliferation- and differentiation-related markers of progression are under investigation that may distinguish non-neoplastic lesions from different neoplastic grades, and could predict the risk of progression to cancer. These findings may have significant influence on novel strategies in cervical cancer diagnosis and screening in the near future. Indeed the continous and deregulated expresson of early HPV viral oncoproteins E6 and E7 have been studied at the nucleic acid level to ascertain cervical infections, and their detection at protein level has been proposed as diagnostic marker [25].

\subsection{Methods of HPV-derived protein detection}

HPV oncoproteins have been mostly detected by immunostaining of cytological or histological preparations, using monoclonal or polyclonal antibodies targeting molecules expressed during the mechanism of cellular transformation and malignancy. Immune responses associated to progressing lesions are preferentially specific early HPV proteins. Biochemical methods detecting oncogenic proteins, such as the early HPV proteins E6 and E7 are in early stages of development as rapid assays for diagnostic purposes.

One E6 detection assay is based on a lateral flow platform, and on the observation that E6 proteins of oncogenic HPVs bind to PDZ proteins [25]. Noteworthy, it has been reported that E6 and E7 proteins of high risk types, such as 16 and 18 , can bind specifically to human cellular proteins containing a PDZ domainprotein in vitro, also known as repeat sequences in the Drosophila discs large protein, the post synaptic density protein PSD.95/SAP 90, and the epithelial tight junction protein $\mathrm{ZO}-1$, hence PDZ. The binding is strong for E6 proteins from oncogenic types as opposed to non-oncogenic types. Thus, it was hypothesized that the interactions between E6 or E7 and PDZ domain proteins could be used as an assay to detect oncogenic E6 expressing cervical lesions, at risk to progression to cancer.

Overexpression of p16INK4a, induced by the viral oncoprotein E7, distinguishes dysplastic lesions from benign changes, and is also being studied and validated as a method to detect advanced lesions [62]. Similarly, PCR primers directed at the E6 or E7 regions, were suggested to be preferable, whereas those targeting the L1 or E1 region can be unreliable and may miss more advanced disease, because the LI/E1 regions are lost during integration of viral DNA into host genomic DNA.

\subsection{Standard reagents for HPV oncogenic proteins and applications}

Standard reagents for use in assays directly detecting cellular oncogenic proteins, virus derived or cell derived, would require the development of specific protein or corresponding antibody standard reagents. The need for such development will depend on the validation and of such tests in clinical settings, and remains to be defined.

\section{Conclusion}

Technology advancements based on detection of viral nucleic acid (sequences), antibodies and cellular biomarkers of disease progression have contributed to changing the landscape of cancer causing infections. Consequently, there is a need to develop quality assurance system for novel HPV products used in a variety of settings for cancer prevention programmes, for disease surveillance, for product improvements, or clinical diagnosis. International standard reagents for HPV would be able to contribute to assure that laboratory services used in evaluation of HPV diseases and preventive interventions are accurate worldwide.

International standard reagents provide a helpful tool for quality assurance of in vitro HPV amplification, detection, genotyping, diagnosis. On one hand, this strategy allows laboratories to measure their results in relation to other groups and improve their performance in HPV detection. High analytical specificity and sensitivity of methods are crucial for both epidemiological surveys and vaccine evaluation, as well as for future monitoring of vaccines performance upon deployment in populations. On the other hand, the usefulness of HPV detection in a diagnostic context should be based 
on its clinical sensitivity and specificity in identifying early disease. The availability of international standards may also allow to compare the various assays, assuring that they have the required sensitivity, so that a negative result assures the safety of a product worldwide.

Once international standard reagents are established, national and local/ or working standards can be derived and calibrated against international units for routine use in the laboratory. It may also be necessary to establish reference panels to aid in the evaluation of diagnostic tests and laboratory proficiency. Indeed, a reference panel of 10 individual genotypes of HIV-1 has been previously established, to help assess the specificity of nucleic acid amplification technology-based assays for HIV-1. ${ }^{2}$

National health authorities need to be prepared to review clinical data, decide on products registration, and control for quality, safety and efficacy of HPV diagnostics, vaccines and therapeutics, for the users within various countries. The national control laboratories need to be prepared to manage the issues related to evaluation of HPV diseases and diagnostic tools, as well as novel HPV vaccines that may arise in the near future. In any event, following approval of any product for general use, quality assurance of HPV antibody measurement kits is important, and national control laboratories should have access to validated reagents to test for quality and eventually for vaccine potency tests, if needed.

Provision of international standard reagents for global use would facilitate the assessment of product and improve the confidence of regulatory authorities on data provided for review. In the mean time, international standard reagents will be useful in determining analytical sensitivity of various methods, in assessing the laboratory performance and in calibrating diagnostic test kits. It is recognized that some international standards may be used for qualitative rather than quantitative purposes.

\section{References}

[1] J.G. Baseman and L.A. Koutsky, The epidemiology of human papillomavirus infections, J Clin Virol 1 (2005), 16-24.

${ }^{2}$ WHO Expert Committee on Biological Standardization, Fifty-third Report 2004, http://www.who.int/biologicals/926-Inside $\% 20$ page.pdf, page 19.
[2] J.J. Carter, M.M. Madeleine, K. Shera, S.M. Schwartz, K.L., Cushing-Haugen, G.C. Wipf, P. Porter, J.R. Daling, J.K. McDougall and D.A. Galloway, Human papillomavirus, 16 and 18 L1 serology compared across anogenital cancer sites, Cancer Res 61 (2001), 1934-1940.

[3] X. Castellsague and N. Munoz, Cofactors in human papillomavirus carcinogenesis-role of parity, oral contraceptives, and tobacco smoking, J Natl Cancer Inst Monogr 31 (2003), 20-28.

[4] P.E. Castle, D. Solomon, M. Schiffman and C.M. Wheeler for the ALTS Group, Human papillomavirus type 16 infections and 2-year absolute risk of cervical precancer in women with equivocal or mild cytologic abnormalities, J Nat Can Inst 97 (2005), 1066-1071.

[5] G.M. Clifford, S. Gallus, R. Herrero, N. Munoz, P.J. Snijders, S. Vaccarella, P.T. Anh, C. Ferreccio, N.T. Hieu, E. Matos, M. Molano, R. Rajkumar, G. Ronco, S. de Sanjose, H.R. Shin, S. Sukvirach, J.O. Thomas, S. Tunsakul, C.J. Meijer and S. Franceschi, IARC HPV Prevalence Surveys Study Group, Worldwide distribution of human papillomavirus types in cytologically normal women in the International Agency for Research on Cancer HPV prevalence surveys: a pooled analysis, Lancet 366 (2005), 991-998.

[6] G.M. Clifford, J.S. Smith, M. Plummer, N. Munoz and S. Franceschi, Human papillomavirus types in invasive cervical cancer worldwide: a meta-analysis, Br J Cancer 88 (2003), $63-73$.

[7] R. Closas Garcia, X. Castellsague, X. Bosch and C.A. Gonzalez, The role of diet and nutrition in cervical carcinogenesis: a review of recent evidence, Int J Cancer 117 (2005), 629-637.

[8] J. Cuzick, A. Szarewski, H. Cubie, G. Hulman, H. Kitchener, D. Luesley, E. McGoogan, U. Menon, G. Terry, R. Edwards, C. Brooks, M. Desai, C. Gie, L. Ho, I. Jacobs, C. Pickles and P. Sasieni, Management of women who test positive for highrisk types of human papillomavirus: the HART study, Lancet 362 (2003), 1871-1876.

[9] D. Dias, J. Van Doren, S. Schlottmann, S. Kelly, D. Puchalski, W. Ruiz, P. Boerckel, J. Kessler, J.M. Antonello, T. Green, M. Brown, J. Smith, N. Chirmule, E. Barr, K.U. Jansen and M.T. Esser, Optimization and validation of a multiplexed luminex assay to quantify antibodies to neutralizing epitopes on human papillomaviruses 6, 11, 16, and 18, Clin Diagn Lab Immunol 8 (2005), 959-969.

[10] K. Elfgren, M. Jacobs, J.M. Walboomers, C.J. Meijer and J. Dillner, Rate of human papillomavirus clearance after treatment of cervical intraepithelial neoplasia, Obstet Gynecol 100 (2002), 965-971.

[11] M. Ferguson, A. Heath, S. Johnes, S. Pagliusi and J. Dillner, Results of the first WHO international collaborative study on the standardization of the detection of antibodies to human papillomaviruses, Int J Cancer 118 (2005), 1508-1514.

[12] D. Galloway, Papillomavirus vaccines in clinical trials, Lancet Infect Dis 8 (2003), 469-475.

[13] L. Gustafsson, J. Pontén, M. Zack and H.-O. Adami, International incidence rates of invasive cervical cancer after introduction of cytological screening, Cancer Causes Cont $\mathbf{8}$ (1997), 755-763.

[14] D.M. Harper, E.L. Franco, C. Wheeler, D.G. Ferris, D. Jenkins, A. Schuind, T. Zahaf, B. Innis, P. Naud, N.S. deCarvalho, C.M. Roteli-Martins, J. Teixeira, M.M. Blattetr, A.P. Korn, W. Quint and G. Dubin, GlaxoSmithKline HPV Vaccine Study Group, Efficacy of a bivalent L1 virus like particle vaccine in prevention of infection with human papillomavirus types 16 
and 18 in young women: a randomized controlled trial, Lancet 364 (2004), 1757-1765.

[15] C.D. Harro, Y.Y.Pang, R.B. Roden, A. Hildesheim, Z. Wang, M.J. ReyNOlds, T.C. Mast, R. Robinson, B.R. Murphy, R.A. Karron, J. Dillner, J.T. Schiller and D. Lowy, Safety and immunogenicity trial in adult volunteers of a human papillomavirus $16 \mathrm{~L} 1$ virus-like particle vaccine, J Natl Cancr Inst 93 (2001), 284-292.

[16] H. zur Hausen, Condylomata acuminata and human genital cancer, Cancer Res 36 (1976), 794

[17] H. zur Hausen, Papillomavirus and cancer: from basic studies to clinical application, Nat Rev Cancer 5 (2002), 342-350.

[18] G.Y. Ho, R. Bierman, L. Beardsley, C.J. Chang and R.D. Burk, Natural history of cervicovaginal papillomavirus infection in young women, $N$ Engl J Med 338 (1998), 423-428.

[19] IARC Monographs on the Evaluation of Carcinogenic Risks to Humans, 2005, http://monographs.iarc.fr/ENG/Meetings/90hpv.pdf.

[20] T. Iftner and L.L. Villa, Chapter 12: Human papillomavirus technologies, J Natl Cancer Inst Monogr 31 (2003), 80-88.

[21] C. Jennings, S.A. Fiscus, S.M. Crowe, A.D. Danilovic, R.J. Morack, S. Scianna, A. Cachafeiro, D.J. Brambilla, J. Schupbach, W. Stevens, R. Respess, O.E. Varnier, G.E. Corrigan, J.S. Gronowitz, M.A. Ussery and J.W. Bremer, Comparison of Two Human Immunodeficiency Virus (HIV) RNA Surrogate Assays to the Standard HIV RNA Assay, J Clin Microbiol 43 (2005), 5950-5956.

[22] A. Josefsson, K. Livak and U. Gyllensten, Detection and quantitation of human papillomavirus by using the fluorescent 5 'exonuclease assay, J Clin Microbiol 37 (1999), 490-496.

[23] J.T. Keating, A. Cviko, S. Riethdorf, L. Riethdorf, B.J. Quade, D. Sun, S. Duensing, E.E. Sheets, K. Munger and C.P. Crum, Ki-67, cyclin E, and p16INK4 are complimentary surrogate biomarkers for human papilloma virus-related cervical neoplasia, Am J Surg Pathol 25 (2001), 884-891.

[24] R. Kirnbauer, Papillomavirus-like particles for serology and vaccine development, Intervirology 39 (1996), 54-61.

[25] T. Kiyono, A. Hiraiwa, M. Fujita, Y. Hayashi, T. Akiyama and M. Ishibashi, Binding of high-risk human papillomavirus E6 oncoproteins to the human homologue of the Drosophila discs large tumor suppressor protein, Proc Natl Acad Sci 94 (1997), 11612-11616.

[26] R. Klaes, T. Friedrich, D. Spitkovsky, R. Ridder, W. Rudy, U. Petry, G. Dallenbach-Hellweg, D. Schmidt and M. von Knebel Doeberitz, Overexpression of p16(INK4A) as a specific marker for dysplastic and neoplastic epithelial cells of the cervix uteri, Cancer Res 59 (1999), 6132-6136.

[27] J.R. Kornegay, M, Roger, P.O. Davies, A.P. Shepard, N.A. Guerrero, B. Lloveras, D. Evans and F. Coutlee, International proficiency study of a consensus L1 PCR assay for the detection and typing of human papillomavirus DNA: evaluation of accuracy and intralaboratory and interlaboratory agreement, $J$ Clin Microbiol 41 (2003), 1080-1086.

[28] L.A. Koutsky, K.A. Ault, C.M. Wheeler, D.R. Brown, E. Barr, F.B. Alvarez, L.M. Chiacchierini and K.U. Jansen, Proof of Principle Study Investigators, A controlled trial of a human papillomavirus type 16 vaccine, N Engl J Med 347 (2002), 1645-1651.

[29] M. Lehtinen, M. Pawlita, K. Zumbach, K. Lie, M. Hakama, E. Jellum, P. Koskela, T. Luostarinen, J. Paavonen, E. Pukkala, E. Sigstad, S. Thoresen and J. Dillner, Evaluation of antibody response to human papillomavirus early proteins in women in whom cervical cancer developed 1 to 20 years later, Am J Obstet Gynecol 188 (2003), 49-55.
[30] A.K. Lie, B. Risberg, B. Borge, B. Sandstad, J. Delabie, R. Rimala, M. Onsrud and S.Thoresen, DNA- versus RNA-based methods for human papillomavirus detection in cervical neoplasia, Gynecol Onco 97 (2005), 908-915.

[31] A. Lorincz and J. Anthony, Advances in HPV detection by Hybrid Capture, Papillomavirus Report 12 (2001), 145-154.

[32] D.R. Lowy and P.M. Howley, Papillomaviruses, in: Fields Virology, D.M. Knipe, P.M. Howley, D.E. Griffin, R.A. Lamb, M.A. Martin, B. Roizman, S.E. Strauss and Pippincott, Chap. 66, $4^{\text {th }}$ edition, eds, Williams \& Wilkins, 2001.

[33] C. Mao, L.A. Koutsky, K.A. Ault,C.M. Wheeler, D.R. Brown, D.J. Wiley, F.B. Alvarez, O.M. Bautista, K.U. Jansen and E. Barr, Efficacy of human papillomavirus-16 vaccine to prevent cervical intraepithelial neoplasia: a randomized controlled trial, Obstet Gynecol 107 (2006), 18-27.

[34] D.J. Marais, J.M. Best, R.C. Rose, P. Keating, R. Soeters, L. Denny, C.M. Dehaeck, J, Nevin, P. Kay, J.A. Passmore and A.L. Williamson, Oral antibodies to human papillomavirus type 16 in women with cervical neoplasia, J Med Virol $\mathbf{6 5}$ (2001), 149-154.

[35] K. Middleton, W. Peh, S. Southern, H. Griffin, K. Sotlar, T. Nakahara, A. El-Sherif, L. Morris, R. Seth, M. Hibma, D. Jenkins, P. Lambert, N. Coleman and J. Doorbar, Organization of human papillomavirus productive cycle during neoplastic progression provides a basis for selection of diagnostic markers, J Virol 77 (2003), 10186-10201.

[36] J. Monsonego, J.M. Bohbot, G. Pollini, C. Krawec, C. Vincent, I. Merignargues, F. Haroun, P. Sednaoui, L. Monfort, R. Dachez and K. Syrjanen, Performance of the Roche AMPLICOR human papillomavirus (HPV) test in prediction of cervical intraepithelial neoplasia (CIN) in women with abnormal PAP smear, Gynecol Onco 99 (2005), 160-168.

[37] N. Munoz, F.X. Bosch, S. de Sanjose, R. Herrero, X. Castellsague, K.V. Shah, P.J. Snijders and C.J. Meijer, International Agency for Research on Cancer Multicenter Cervical Cancer Study Group, Epidemiologic classification of human papillomavirus types associated with cervical cancer, $N$ Engl J Med 348 (2003), 518-527.

[38] D. Nardelli-Haefliger, D. Wirthner, J.T. Schiller, D.R. Lowy, A. Hildesheim, F. Ponci and P. De Grandi, Specific antibody levels at the cervix during the menstrual cycle of women vaccinated with human papillomavirus 16 virus-like particles, $J$ Natl Cancer Inst 95 (2003), 1128-1137.

[39] M.A. Nobbenhuis, T.J. Helmerhorst, A.J. van den Brule, L. Rozendaal, P.D. Bezemer, F.J. Voorhorst and C.J. Meijer, High-risk human papillomavirus clearance in pregnant women: trends for lower clearance during pregnancy with a catchup postpartum, Br J Cancer 87 (2002), 75-80.

[40] P. Ohlschlager, W. Osen, K. Dell, S. Faath, R.L. Garcea, I. Jochmus, M. Muller, M. Pawlita, K. Schafer, P. Sehr, C. Staib, G. Sutter and L. Gissmann, Human papillomavirus type 16 L1 capsomeres induce L1-specific cytotoxic T lymphocytes and tumor regression in C57BL/6 mice, J Virol 77 (2003), 4635-4645.

[41] D. Opalka, C.E. Lachman, S.A. MacMullen, K.U. Jansen, J.F. Smith, N. Chirmule and M.T. Esser, Simultaneous quantitation of antibodies to neutralizing epitopes on virus-like particles for human papillomavirus types $6,11,16$, and 18 by a multiplexed luminex assay, Clin Diagn Lab Immunol 10 (2003), 108-115.

[42] A.G. Östör, Natural history of cervical intraepithelial neoplasia: A critical review, Int J Gynecol Pathol 12 (1993), 86-92.

[43] G.N. Papanicolaou and H.F. Traut, The diagnostic value of vaginal smears in carcinoma of uterus, Am J Obstet Gynecol 42 (1941), 193-205. 
[44] D.M. Parkin, The global health burden of infection-associated cancers in the year 2002, Int J Cancer 118 (2006), 3030-3044.

[45] D.M. Parkin, F. Bray, J. Ferlay and P. Pisani, Global cancer statistics 2002, CA Cancer J Clin 55 (2005), 74-108.

[46] D.V. Pastrana, C.B. Buck, Y.Y. Pang, C.D. Thompson, P.E. Castle, P.C. FitzGerald, S. Kruger Kjaer, D.R. Lowy and J.T. Schiller, Reactivity of human sera in a sensitive, highthroughput pseudovirus-based papillomavirus neutralization assay for HPV16 and HPV18, Virology 321 (2004), 205-216.

[47] W. Quint, S.R. Pagliusi, N. Lelie, E.-M. de Villiers, C.M. Wheeler and the World Health Organization Human Papillomavirus DNA International Collaborative Study Group, Results of the First World Health Organization International Collaborative Study of Detection of Human Papillomavirus DNA, J Clin Microbiol 44 (2006), 571-579.

[48] R.B. Roden, N.L. Hubbert, R. Kirnbauer, N.D. Christensen, D.R. Lowy and J.T. Schiller, Assessment of the serological relatedness of genital human papillomaviruses by hemagglutination inhibition, J Virol 70 (1996), 3298-3301.

[49] J. Saldanha, A. Heath, N. Lelie, G. Pisani and M.Y. Yu, Collaborative Study Group, A World Health Organization International Standard for hepatitis A virus RNA nucleic acid amplification technology assays, Vox Sang 89 (2005), 52-58.

[50] J.T. Schiller, Papillomavirus-like particle vaccines for cervical cancer, Mol Med Today 5 (1999), 209-215.

[51] J. Schirm, A.M. van Loon, E. Valentine-Thon, P.E. Klapper, J. Reid and G.M. Cleator, External quality assessment program for qualitative and quantitative detection of hepatitis $\mathrm{C}$ virus RNA in diagnostic virology, J Clin Microbiol 40 (2002), 29732980.

[52] N.F. Schlecht, Prognostic value of human papillomavirus in the survival of head and neck cancer patients: an overview of the evidence, Oncol Rep 14 (2005), 1239-1247.

[53] M. Schmitt, I.G. Bravo, P.J. Snijders, L. Gissmann, M. Pawlita and T. Waterboer, Bead-based multiplex genotyping of human papillomaviruses, J Clin Microbiol 44 (2006), 504-512.

[54] P. Sehr, M. Muller, R. Hoepfl, A. Widschwendter and M Pawlita, HPV antibody detection by ELISA with capsid protein L1 fused to glutathione S-transferase, $J$ Virol Meth 106 (2002), 61-70.

[55] S.S. Seo, Y.S. Song, J.W. Kim, N.H. Park, S.B. Kang and H.P. Lee, Good correlation of HPV DNA test between selfcollected vaginal and clinician-collected cervical samples by the oligonucleotide microarray, Gynecol Oncol 102 (2006), 67-73.

[56] H.R. Shin, S. Franceschi, S. Vaccarella, J.W. Roh, Y.H. Ju, J.K. Oh, H.J. Kong, S.H. Rha, S.I. Jung, J.I. Kim, K.Y. Jung, L.J. van Doorn and W. Quint, Prevalence and determinants of genital infection with papillomavirus, in female and male university students in Busan, South Korea, J Infect Dis 190 (2004), 468-476.

[57] I. Silins, E. Avall-Lundqvist, A. Tadesse, K.U. Jansen, U. Stendahl, P. Lenner, K. Zumbach, M. Pawlita, J. Dillner and B. Frankendal, Evaluation of antibodies to human papillomavirus as prognostic markers in cervical cancer patients, Gynecol Oncol 85 (2002), 333-338.

[58] J.S. Smith, C. Bosetti, N. Munoz, R. Herrero, F.X. Bosch, J. Eluf-Neto, C.J. Meijer, A.J. Van Den Brule, S. Franceschi and R.W. Peeling, IARC multicentric case-control study, Chlamydia trachomatis and invasive cervical cancer: a pooled analysis of the IARC multicentric case-control study, Int J Cancer 111 (2004), 431-439.
[59] P.J. Snijders, A.J. van den Brule and C.J. Meijer, The clinical relevance of human papillomavirus testing: relationship between analytical and clinical sensitivity, J Pathol 201 (2003), $1-6$.

[60] Technical workshop on international collaborative studies on HPV reagents for laboratory diagnostic procedures: a progress report. http://www.who.int/vaccines-documents/ DocsPDF04/www786.pdf.

[61] L.L. Villa, R.L. Costa, C.A. Petta, R.P. Andrade, K.A. Ault, A.R. Giuliano, C.M. Wheeler, L.A. Koutsky, C. Malm, M. Lehtinen, F.E. Skjeldestad, S.E. Olsson, M. Steinwall, D.R. Brown, R.J. Kurman, B.M. Ronnett, M.H. Stoler, A. Ferenczy, D.M. Harper, G.M. Tamms, J. Yu, L. Lupinacci, R. Railkar, F.J. Taddeo, K.U. Jansen, M.T. Esser, H.L. Sings, A.J. Saah and E.Barr, Prophylactic quadrivalent human papillomavirus (types $6,11,16$, and 18) $\mathrm{L} 1$ virus-like particle vaccine in young women: a randomised double-blind placebo-controlled multicentre phase II efficacy trial, Lancet Oncol 6 (2005), 271-278.

[62] S. Vinokurova, N. Wentzensen and M. von Knebel Doeberitz, Analysis of p16INK4a and integrated HPV genomes as progression markers, Methods Mol Med 119 (2005), 73-83.

[63] J.M. Walboomers, M.V. Jacobs, M.M. Manos, F.X. Bosch, J.A. Kummer, K.V. Shah, P.J. Snijders, J. Peto, C.J. Meijer and N. Munoz, Human papillomavirus is a necessary cause of invasive cervical cancer worldwide, J Pathol 189 (1999), $12-19$.

[64] X. Wang, M. Sapp, N.D. Christensen and J. Dillner, Heparinbased ELISA reduces background reactivity in virus-like particle-based papillomavirus serology, J Gen Virol 85 (2005), 65-73.

[65] T. Waterboer, P. Sehr, K.M. Michael, S. Franceschi, J.D. Nieland, T.O. Joos, M.F. Templin and M. Pawlita, Multiplex human papillomavirus serology based on in situ-purified glutathione s-transferase fusion proteins, Clin Chem 51 (2005), $1845-1853$.

[66] T. Waterboer, P. Sehr and M. Pawlita, Suppression of nonspecific binding in serological Luminex assays, J Immunol Methods 309 (2005), 200-204.

[67] N. Wentzensen, C. Bergeron, F. Cas, D. Eschenbach, S. Vinokurova and M. von Knebel Doeberitz, Evaluation of a nuclear score for p16INK4a-stained cervical squamous cells in liquid-based cytology samples, Cancer 105 (2005), 461-467.

[68] WHO Expert Committee on Biological Standardization, Fifty-third Report, http://www.who.int/biologicals/926Inside\%20page.pdf.

[69] WHO informal workshop: development of international HPV reference reagents, 2001. http://www.who.int/vaccinesdocuments/DocsPDF02/www699.pdf.

[70] WHO Technical workshop on cellular mediated immunity to human papillomavirus, 2005. http://www.who.int/vacciesdocuments/DocsPDF06/824scxreen.pdf.

[71] WHO Technical Report Series http://whqlibdoc.who.int/trs/ WHO_TRS_897.pdf.

[72] T.C. Wright, L. Denny, L. Kuhn, A. Pollack and A. Lorincz, HPV DNA testing of self-collected vaginal samples compared with cytologic screening to detect cervical cancer, JAMA 283 (2000), 81-86

[73] T.C. Wright, M. Schiffman, D. Solomon, J. Cox, F. Garcia, S. Goldie, K. Hatch, K. Noller, N. Roach, C. Runowicz and D. Saslow, Interim guidance for the use of human papillomavirus DNA testing as an adjunct to cervical cytology for screening, Obstet Gynecol 103 (2004), 304-309. 
[74] N. Ylitalo, P. Sorensen, A.M. Josefsson, P.K. Magnusson, P.K. Andersen, J. Ponten, H.O. Adami, U.B. Gyllensten and M. Melbye, Consistent high viral load of human papillomavirus
16 and risk of cervical carcinoma in situ: a nested case-control study, Lancet 355 (2000), 2194-2198. 


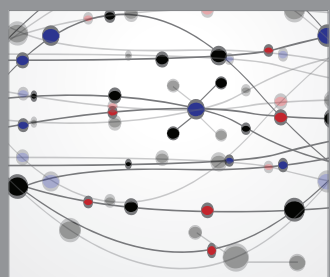

The Scientific World Journal
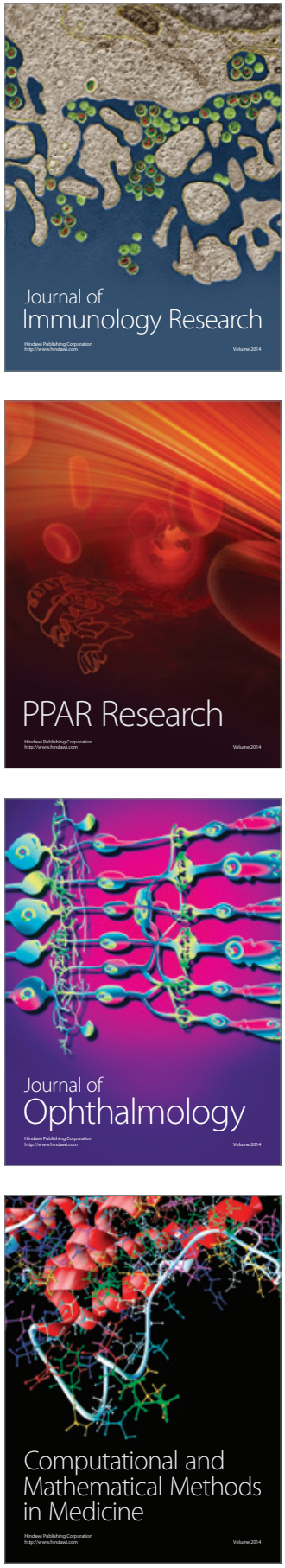

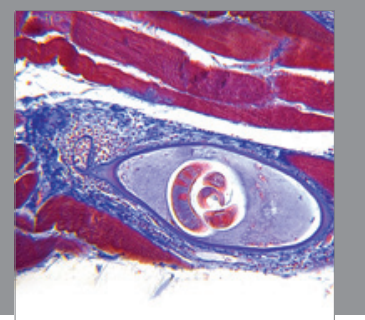

Gastroenterology

Research and Practice
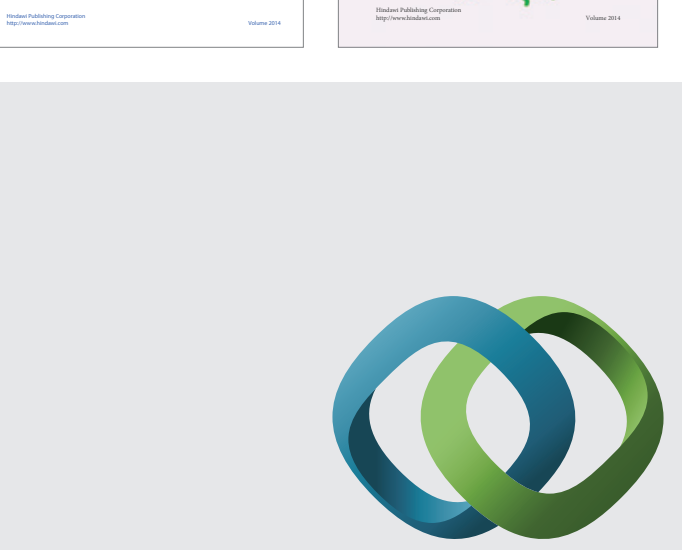

\section{Hindawi}

Submit your manuscripts at

http://www.hindawi.com
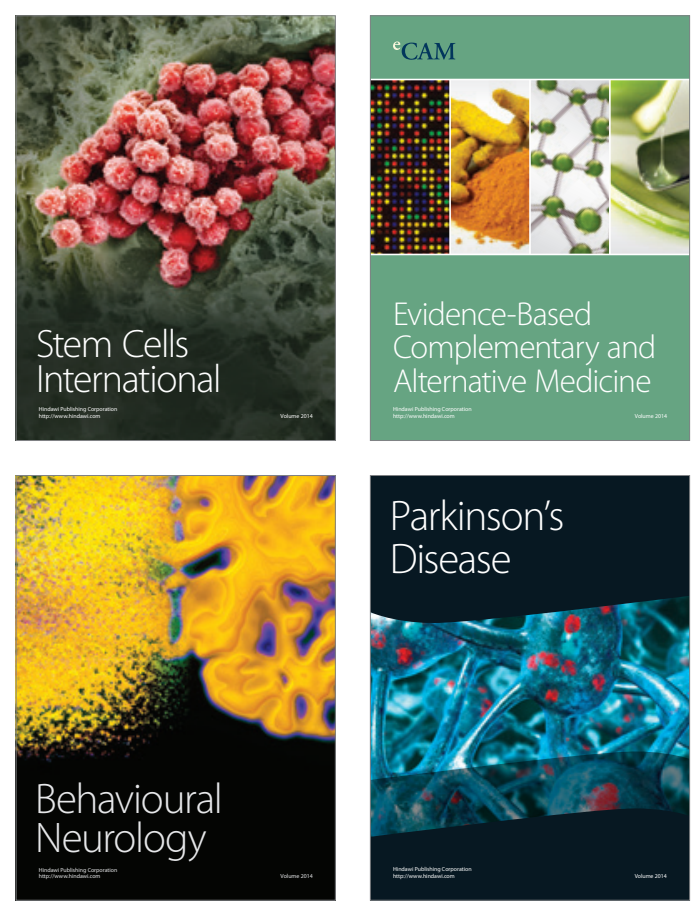

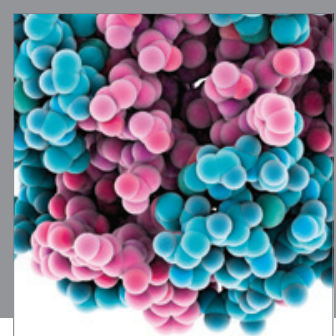

Journal of
Diabetes Research

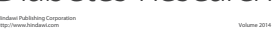

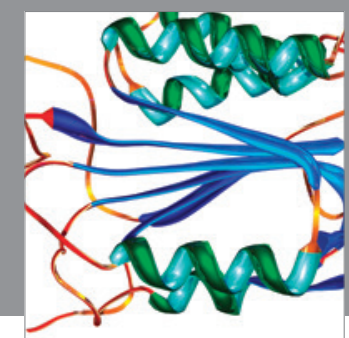

Disease Markers
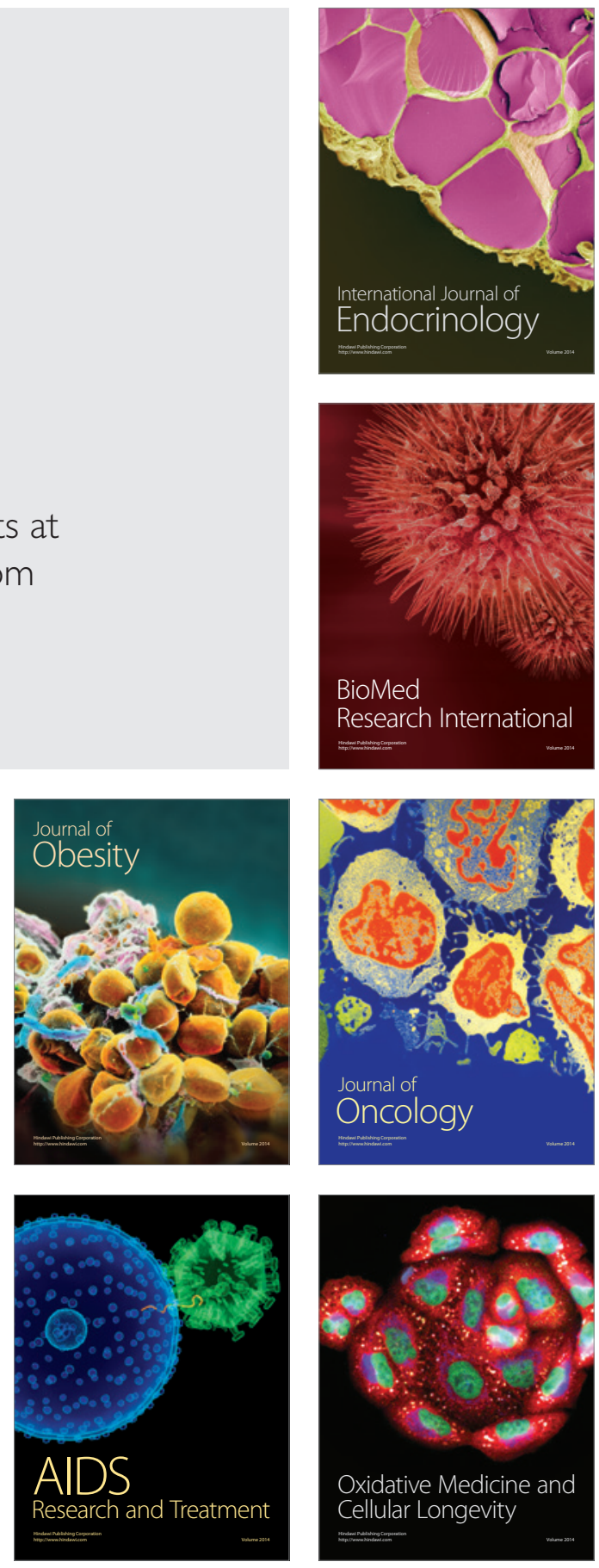